\title{
Erratum to: FCGR3A-158 polymorphism influences the biological response to infliximab in Crohn's disease through affecting the ADCC activity
}

\author{
Rintaro Moroi ${ }^{1,3} \cdot$ Katsuya Endo $^{1} \cdot$ Yoshitaka Kinouchi $^{1}$. \\ Hisashi Shiga ${ }^{1}$ - Yoichi Kakuta ${ }^{1}$ Masatake Kuroha ${ }^{1}$. \\ Yoshitake Kanazawa $^{1}$ • Yosuke Shimodaira ${ }^{1}$. \\ Takahiko Horiuchi $^{2} \cdot$ Seiichi Takahashi ${ }^{1}$. \\ Tooru Shimosegawa ${ }^{1}$
}

Published online: 6 August 2015

(C) Springer-Verlag Berlin Heidelberg 2015

Erratum to: Immunogenetics (2013) 65:265-271

DOI 10.1007/s00251-013-0679-8

In response to an enquiry regarding a possible discrepancy in our original Table 2 data, we - the authors of the paper - recalculated the material. We were able to confirm that our original calculation of the raw data - which had excluded certain missing values - was appropriate, but in recalculating we found minor errors in the Table 2 values. We have corrected these. A revised Table 2, with new values indicated in boldface, is below.

The corrections do not affect any statistical differences among the genotypes or any of the article's results and conclusions.

The online version of the original article can be found at http://dx.doi.org/ 10.1007/s00251-013-0679-8.

Rintaro Moroi

rinta@med.tohoku.ac.jp

1 Division of Gastroenterology, Tohoku University Graduate School of Medicine, Aoba-ku, Sendai, Japan

2 Department of Medicine and Biosystemic Science, Kyushu University Faculty of Medicine, Kyushu, Japan

3 1-1, Seiryo, Aoba-ku, Sendai, Miyagi, Japan 